\title{
ABSOLUTE STABILITY OF A CLASS OF FRACTIONAL POSITIVE NONLINEAR SYSTEMS
}

\author{
TADEUSZ KACZOREK ${ }^{a}$
}

${ }^{a}$ Faculty of Electrical Engineering

Białystok University of Technology, Wiejska 45D, 15-351 Białystok, Poland

e-mail: kaczorek@ee.pw.edu.pl

\begin{abstract}
The positivity and absolute stability of a class of fractional nonlinear continuous-time and discrete-time systems are addressed. Necessary and sufficient conditions for the positivity of this class of nonlinear systems are established. Sufficient conditions for the absolute stability of this class of fractional positive nonlinear systems are also given.
\end{abstract}

Keywords: absolute stability, fractional system, positive system, nonlinear system.

\section{Introduction}

A dynamical system is called positive if its trajectory starting from any nonnegative initial state remains forever in the positive orthant for all nonnegative inputs. An overview of the state of the art in positive theory is given by Berman and Plemmons (1994), Farina and Rinaldi (2000) or Kaczorek (2002). A variety of models having positive behavior can be found in engineering, economics, social sciences, biology and medicine, etc. (cf. Ait Rami and Tadeo, 2007; Ortigueira, 2011; Zhang et al., 2014; Xiang-Jun, 2008).

Mathematical fundamentals of fractional calculus are given by Oldham and Spanier (1974), Ortigueira (2011), Ostalczyk (2008) and Podlubny (1999). Positive fractional linear systems were investigated by Kaczorek (2018; 2011b) and Ostalczyk (2008). The stability of linear and nonlinear standard and positive fractional systems was addressed by Busłowicz (2008; 2012), Busłowicz and Kaczorek (2009), Farina and Rinaldi (2000), Kaczorek (2016; 2015a; 2015b; 2011b), Ortigueira (2011), Ostalczyk (2008), Podlubny (1999) as well as Polyak and Shcherbakov (2002a). The stabilization of positive descriptor fractional systems was investigated by Kaczorek (2018; 2014), Ortigueira (2011) and Ostalczyk (2008). Superstable linear systems have been addressed by Polyak and Shcherbakov (2002a; 2002b). Positive linear systems with different fractional orders were introduced by Kaczorek (2010; 2011a), while their stability was analyzed by Busłowicz (2008; 2012) and Ortigueira (2011). The notion of practical stability of positive fractional linear systems was introduced by Kaczorek (2002). Some recent interesting results in fractional systems theory and its applications can be found in the works of Berman and Plemmons (1994), Kaczorek (2010), Zhang et al. (2014) and Xiang-Jun (2008).

In this paper, the positivity and absolute stability of a class of nonlinear continuous-time and discrete-time systems will be investigated. The paper is organized as follows. In Section 2, some preliminaries concerning the positivity and stability of linear systems are recalled. The positivity and absolute stability of fractional positive continuous-time nonlinear systems are investigated in Section 3 and these of fractional positive discrete-time nonlinear systems in Section 4. Concluding remarks are given in Section 5 .

The following notation will be used: $\mathbb{R}$, the set of real numbers; $\mathbb{R}^{n \times m}$, the set of $n \times m$ real matrices; $\mathbb{R}_{+}^{n \times m}$, the set of $n \times m$ real matrices with nonnegative entries and $\mathbb{R}_{+}^{n}=\mathbb{R}_{+}^{n \times 1} ; M_{n}$, the set of $n \times n$ Metzler matrices (real matrices with nonnegative off-diagonal entries); $I_{n}$, the $n \times n$ identity matrix; $A^{T}$, the transpose of matrix $A$.

\section{Preliminaries}

The following Caputo definition of the fractional derivative will be used (Kaczorek, 2011b; Oldham and Spanier, 1974; Ortigueira, 2011; Ostalczyk, 2008; 
Podlubny, 1999):

$$
\begin{aligned}
\frac{\mathrm{d}^{\alpha}}{\mathrm{d} t^{\alpha}} f(t) & =\frac{1}{\Gamma(n-\alpha)} \int_{0}^{f} \frac{f^{(n)}}{(t-\tau)^{\alpha+1-n}} \mathrm{~d} \tau, \\
n-1 & <\alpha \leq n \in \mathbb{N}=\{1,2, \ldots\},
\end{aligned}
$$

where $\alpha \in \mathbb{R}$ is the order of the fractional derivative and

$$
f^{(n)}(\tau)=\frac{\mathrm{d}^{n} f(\tau)}{\mathrm{d} \tau^{n}},
$$

while $\Gamma(x)=\int_{0}^{\infty} e^{-t} t^{x-1} \mathrm{~d} t$ is the gamma function.

Consider a fractional linear system described by the equations

$$
\begin{aligned}
\frac{\mathrm{d}^{\alpha} x}{\mathrm{~d} t^{\alpha}} & =A x+B u, \quad 0<\alpha \leq 1, \\
y & =C x,
\end{aligned}
$$

where $x=x(t) \in \mathbb{R}^{n}, u=u(t) \in \mathbb{R}^{m}, y=y(t) \in \mathbb{R}^{p}$ are the state, input and output vectors, respectively, and $A \in \mathbb{R}^{n \times n}, B \in \mathbb{R}^{n \times m}, C \in \mathbb{R}^{p \times n}$

Definition 1. (Kaczorek, 2011b) The fractional system (2) is called an (internally) positive system if and only if $x(t) \in \mathbb{R}_{+}^{n}$ and $y(t) \in \mathbb{R}_{+}^{p}$ for $t \geq 0$ for any initial conditions $x_{0} \in \mathbb{R}_{+}^{n}$ and all inputs $u(t) \in \mathbb{R}_{+}^{m}, t \geq 0$.

Theorem 1. (Kaczorek, 2011b) The continuous-time fractional system (2) is (internally) positive if and only if the matrix $A$ is a Metzler matrix and

$$
A \in M_{n}, \quad B \in \mathbb{R}_{+}^{n \times m}, \quad C \in \mathbb{R}_{+}^{p \times n} .
$$

Definition 2. (Kaczorek, 2011c) The positive fractional system (2) is called asymptotically stable if

$$
\lim _{x \rightarrow \infty} x(t)=0, \quad \forall x(0) \in \mathbb{R}_{+}^{n} .
$$

Theorem 2. (Kaczorek, 2011b) The positive fractional system (2) is asymptotically stable if and only if one of the following equivalent conditions is satisfied:

(i) all the coefficients of the characteristic polynomial

$$
\begin{aligned}
p_{n}(s) & =\operatorname{det}\left[I_{n} s-A\right] \\
& =s^{n}+a_{n-1} s^{n-1}+\cdots+a_{1} s+a_{0}
\end{aligned}
$$

are positive, i.e., $a_{i}>0$ for $i=0,1, \ldots, n-1$;

(ii) there exists a strictly positive vector $\lambda^{T}=$ $\left[\begin{array}{lll}\lambda_{1} & \cdots & \lambda_{n}\end{array}\right]^{T}, \lambda_{k}>0, k=1, \ldots, n$, such that

$$
A \lambda<0 \quad \text { or } \quad \lambda^{T} A<0 .
$$

If the matrix $A$ is nonsingular, then we can choose $\lambda=A^{-1} c$, where $c \in \mathbb{R}^{n}$ is strictly positive.
Consider the fractional discrete-time linear system (Kaczorek, 2011b; Ostalczyk, 2008)

$$
\begin{aligned}
\Delta^{\alpha} x_{i+1} & =A x_{i}+B u_{i}, \quad i \in \mathbb{Z}_{+}=\{0,1, \ldots\}, \\
y_{i} & =C x_{i}, \\
\Delta^{\alpha} x_{i} & =\sum_{j=0}^{i} c_{j} x_{i-j}, c_{j}=(-1)^{j}\left(\begin{array}{c}
\alpha \\
j
\end{array}\right) \\
& = \begin{cases}1 & \text { for } j=0, \\
(-1)^{j} \frac{\alpha(\alpha-1) \cdots(\alpha-j+1)}{j !} & \text { for } j=1,2, \ldots,\end{cases}
\end{aligned}
$$

$x_{i} \in \mathbb{R}^{n}, u_{i} \in \mathbb{R}^{m}, y_{i} \in \mathbb{R}^{p}$ being the state, input and output vectors, respectively, and $A \in \mathbb{R}^{n \times n}, B \in \mathbb{R}^{n \times m}$, $C \in \mathbb{R}^{p \times n}$

Definition 3. (Farina and Rinaldi, 2000; Kaczorek, $2011 b$; 2002) The fractional discrete-time linear system (7) is called (internally) positive if $x_{i} \in \mathbb{R}_{+}^{n}, y_{i} \in \mathbb{R}_{+}^{p}$, $i \in \mathbb{Z}_{+}$, for any initial conditions $x_{0} \in \mathbb{R}_{+}^{n}$ and all inputs $u_{i} \in \mathbb{R}_{+}^{m}, i \in \mathbb{Z}_{+}$.

Theorem 3. (Farina and Rinaldi, 2000; Kaczorek, 2002) The fractional discrete-time linear system (7) is positive if and only if

$$
A \in \mathbb{R}_{+}^{n \times n}, \quad B \in \mathbb{R}_{+}^{n \times m}, \quad C \in \mathbb{R}_{+}^{p \times n} .
$$

Definition 4. (Farina and Rinaldi, 2000; Kaczorek, $2011 b$; 2002) The fractional positive discrete-time system (7) is called asymptotically stable if

$$
\lim _{i \rightarrow \infty} x_{i}=0, \quad \forall x_{0} \in \mathbb{R}_{+}^{n} .
$$

Theorem 4. (Farina and Rinaldi, 2000; Kaczorek, 2011b; 2002) The fractional positive discrete-time linear system (7) is asymptotically stable if and only if one of the following equivalent conditions is satisfied:

(i) all the coefficients of the characteristic polynomial

$$
\begin{aligned}
p_{n}(z) & =\operatorname{det}\left[I_{n}(z+1)-A\right] \\
& =z^{n}+a_{n-1} z^{n-1}+\cdots+a_{1} z+a_{0}
\end{aligned}
$$

are positive, i.e., $a_{i}>0$ for $i=0,1, \ldots, n-1$;

(ii) there exists a strictly positive vector $\lambda^{T}=$ $\left[\begin{array}{lll}\lambda_{1} & \cdots & \lambda_{n}\end{array}\right]^{T}, \lambda_{k}>0, k=1, \ldots, n$ such that

$$
\left(A-I_{n}\right) \lambda<0 \quad \text { or } \quad \lambda^{T}\left(A^{T}-I_{n}\right)<0 .
$$

If the matrix $\left(A-I_{n}\right)$ is nonsingular then we can choose $\lambda=\left(A-I_{n}\right)^{-1} c$, where $c \in \mathbb{R}^{n}$ is strictly positive. 


\section{Absolute stability of positive continuous-time nonlinear systems}

Consider the fractional nonlinear continuous-time system shown in Fig. 1 and described by the equations

$$
\begin{aligned}
\frac{\mathrm{d}^{\alpha} x}{\mathrm{~d} t^{\alpha}} & =A x+B u, \quad u=f(e), \quad 0<\alpha<1, \\
y & =C x
\end{aligned}
$$

where $x=x(t) \in \mathbb{R}^{n}, u=u(t) \in \mathbb{R}^{m}, y=y(t) \in \mathbb{R}^{p}$ are respectively the state, input and output vectors of the system $A \in \mathbb{R}^{n \times n}, B \in \mathbb{R}^{n \times 1}, C \in \mathbb{R}^{1 \times n}$, and the characteristic $f(e)$ of the nonlinear element (Fig. 2) satisfies the conditions

$$
\begin{gathered}
k e<f(e)<0, \quad \text { if } \quad e<0, \\
0<f e<k e \text { if } \quad e>0, \\
f(0)=0 .
\end{gathered}
$$

Definition 5. The fractional nonlinear system (12) is called (internally) positive if $x(t) \in \mathbb{R}_{+}^{n}, y(t) \in \mathbb{R}_{+}^{p}$, $t \geq 0$ for any initial conditions $x(0) \in \mathbb{R}_{+}^{n}$ and all inputs $u(t) \in \mathbb{R}_{+}, t \geq 0$.

Theorem 5. The fractional nonlinear system (12) is positive if and only if

$$
A \in M_{n}, \quad B \in \mathbb{R}_{+}^{n \times 1}, \quad C \in \mathbb{R}_{+}^{1 \times n},
$$

and the conditions (13) are satisfied.

Proof. It is well known (Kaczorek, 2011b; Bartosiewicz, 2017) that, if $u=f(e) \geq 0, t \geq 0$, then $x(t) \in \mathbb{R}_{+}^{n}, t \geq 0$ for $x(0) \in \mathbb{R}_{+}^{n}$ if and only if $A \in M_{n}$ and $B \in \mathbb{R}_{+}^{n \times 1}$. From (12b) for $t=0$ we have $y(0)=C x(0) \in \mathbb{R}_{+}$for $x(0) \in \mathbb{R}_{+}^{n}$ if and only if $C \in \mathbb{R}_{+}^{1 \times n}$.

Definition 6. The fractional positive nonlinear system (12) is called absolutely stable if $x(t) \in \mathbb{R}_{+}^{n}, t \geq 0$, and

$$
\lim _{t \rightarrow \infty} x(t)=0, \quad \forall x(0) \in \mathbb{R}_{+}^{n} .
$$

The Metzler matrix $A \in M_{n}$ is called a Hurwitz Metzler matrix if all its eigenvalues $\lambda_{k}$ satisfy the condition $\mathbb{R} \lambda_{k}<0, \quad k=1, \ldots, n$.

Theorem 6. The positive fractional nonlinear system (12) is absolutely stable if

(i) $A \in M_{n}$ is the Hurwitz Metzler matrix,

$$
B \in \mathbb{R}_{+}^{n \times 1}, \quad C \in \mathbb{R}_{+}^{1 \times n}
$$

(ii) the nonlinear characteristic $f(e)$ satisfies the condition (13).

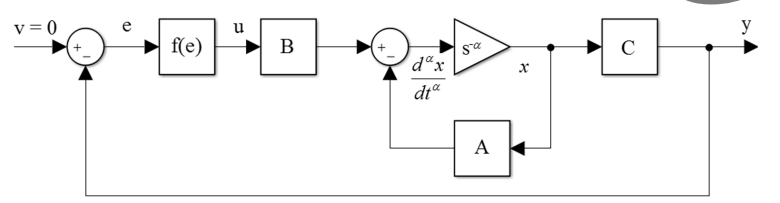

Fig. 1. Fractional nonlinear continuous-time system.

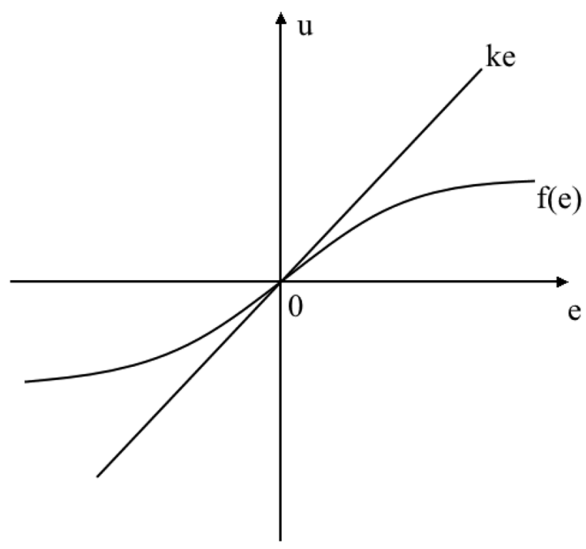

Fig. 2. Characteristic $f(e)$ of the nonlinear element.

Proof. The proof is based on the Lyapunov method for fractional positive systems. As a candidate for the Lyapunov function the following linear function of the state vector $x(t) \in \mathbb{R}_{+}^{n}, t \geq 0$, is assumed:

$$
\begin{aligned}
V(x(t)) & =\lambda^{T} x(t), \\
\lambda^{T} & =\left[\begin{array}{lll}
\lambda_{1} & \ldots & \lambda_{n}
\end{array}\right], \\
\lambda_{k} & >0, \quad k=1, \ldots, n .
\end{aligned}
$$

Using (17) and (12a), we obtain

$$
\begin{aligned}
\frac{\mathrm{d}^{\alpha} V(x)}{\mathrm{d} t^{\alpha}} & =\lambda^{T} \frac{\mathrm{d}^{\alpha} x(t)}{\mathrm{d} t^{\alpha}} \\
& =\lambda^{T}[A x(t)+B f(e)]<0
\end{aligned}
$$

since, by (16) and (6),

$$
\lambda^{T} A<0 \text { and } f(-e)<0 \text { for }-e<0 \text { and } t \geq 0 \text {. }
$$

Therefore, the fractional positive nonlinear system (12) is absolutely stable if both the conditions of Theorem 6 are satisfied.

Remark 1. The absolute stability of the fractional positive nonlinear system is directly independent of the transfer function of its linear part (and also of its fractional frequency characteristics).

Example 1. Consider the fractional nonlinear system (11) with

$$
\begin{aligned}
& A=\left[\begin{array}{cc}
-2 & 1 \\
1 & -3
\end{array}\right], \quad B=\left[\begin{array}{l}
1 \\
0
\end{array}\right] \\
& C=\left[\begin{array}{ll}
0 & 2
\end{array}\right] .
\end{aligned}
$$


The fractional positive nonlinear system is absolutely stable for the characteristic $f(e)$ satisfying the condition (13) since the matrix $A \in M_{2}$ is asymptotically stable and its characteristic polynomial

$$
\operatorname{det}\left[I_{2} s-A\right]=\left|\begin{array}{cc}
s+2 & -1 \\
-1 & s+3
\end{array}\right|=s^{2}+5 s+5
$$

has positive coefficients (Theorem 2, Condition (i)).

The same result follows from Condition (ii) of Theorem 2 since, for

$$
\lambda=\left[\begin{array}{l}
1 \\
1
\end{array}\right]
$$

we have

$$
A \lambda=\left[\begin{array}{cc}
-2 & 1 \\
1 & -3
\end{array}\right]\left[\begin{array}{l}
1 \\
1
\end{array}\right]=\left[\begin{array}{l}
-1 \\
-2
\end{array}\right]<0 .
$$

Therefore, by Theorem 6 the fractional nonlinear system with 20 is absolutely stable for all nonlinear characteristics $f(e)$ satisfying the condition (13).

\section{Absolute stability of fractional discrete-time nonlinear systems}

Consider a fractional nonlinear discrete-time system shown in Fig. 3 and described by the equations

$$
\begin{aligned}
\Delta^{\alpha} x_{i+1} & =A x_{i}+B u_{i}, \\
y_{i} & =C x_{i}, \\
u_{i} & =f\left(e_{i}\right), \quad i \in \mathbb{Z}_{+}=\{0,1, \ldots\}
\end{aligned}
$$

where $x_{i} \in \mathbb{R}^{n}, u_{i} \in \mathbb{R}^{m}, y_{i} \in \mathbb{R}$ are respectively the state, input and output vectors of the system $A \in \mathbb{R}^{n \times n}$, $B \in \mathbb{R}^{n \times 1}, C \in \mathbb{R}^{1 \times n}$, and the characteristic $f\left(e_{i}\right)$ of the nonlinear element (Fig. 4) satisfies the condition

$$
0<f\left(e_{i}\right)<k e_{i}, \quad 0<k<\infty,
$$

and

$$
\begin{gathered}
\Delta^{\alpha} x_{i}=\sum_{j=0}^{i}(-1)^{j}\left(\begin{array}{c}
\alpha \\
j
\end{array}\right) x_{i-j}, \quad 0<\alpha<1, \\
\left(\begin{array}{c}
\alpha \\
j
\end{array}\right)= \begin{cases}1 & \text { for } j=0, \\
\frac{\alpha(\alpha-1) \cdots(\alpha-j+1)}{j !} & \text { for } j=1,2, \ldots\end{cases}
\end{gathered}
$$

is the fractional $\alpha$-th order difference of $x_{i}$.

Substitution of (25) into (23a) yields

$$
\begin{aligned}
x_{i+1} & +\sum_{j=2}^{i+1} c_{j} x_{i-j+1} \\
& =A_{\alpha} x_{i}+\operatorname{Bf}\left(e_{i}\right), \quad i \in \mathbb{Z}_{+},
\end{aligned}
$$

where

$$
A_{\alpha}=A+\mathrm{I}_{n} \alpha, \quad C_{j}=(-1)^{j}\left(\frac{\alpha}{j}\right) .
$$

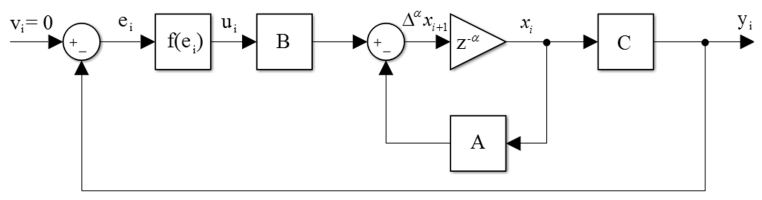

Fig. 3. Fractional nonlinear discrete-time system.

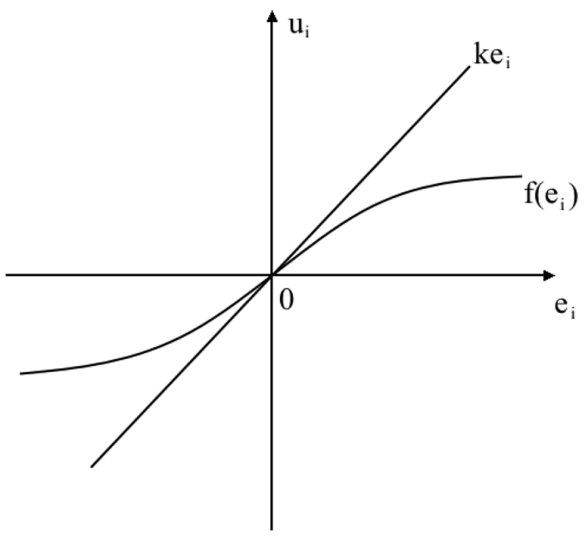

Fig. 4. Characteristic $f\left(e_{i}\right)$ of the nonlinear element.

Definition 7. The fractional nonlinear system (23) is called (internally) positive if $x_{i} \in \mathbb{R}_{+}^{n}, y_{i} \in \mathbb{R}_{+}, i \in \mathbb{Z}_{+}$, for every initial condition $x_{0} \in \mathbb{R}_{+}^{n}$ and all inputs $u_{i} \in$ $\mathbb{R}_{+}, i \in \mathbb{Z}_{+}$.

Theorem 7. The fractional nonlinear system (23) is positive if and only if

$$
A_{\alpha} \in \mathbb{R}_{+}^{n \times n}, \quad B \in \mathbb{R}_{+}^{n \times 1}, \quad C \in \mathbb{R}_{+}^{1 \times n},
$$

and

for all $i \in \mathbb{Z}_{+}$

$$
\begin{aligned}
f\left(e_{i}\right) & \geq 0 \quad \text { for } \quad e_{i} \geq 0, \\
f\left(-e_{i}\right) & <0 \quad \text { for }-e_{i}<0
\end{aligned}
$$

Proof. It is well known (Kaczorek, 2011b) that, if $u_{i}=$ $f\left(e_{i}\right) \geq 0, i \geq 0$, then $x_{i} \in \mathbb{R}_{+}^{n}, i \in \mathbb{Z}_{+}$, for every $x_{i} \in \mathbb{R}_{+}^{n}$ if and only if $A_{\alpha} \in \mathbb{R}_{+}^{n \times n}$ and $B \in \mathbb{R}_{+}^{n \times 1}$ since $c_{j}>0$ for $j=1,2, \ldots$. From (23b), for $i=0$ we have $y_{0}=C x_{0} \in \mathbb{R}_{+}$for $x_{0} \in \mathbb{R}_{+}^{n}$ if and only if $C \in \mathbb{R}_{+}^{1 \times n}$.

Definition 8. The fractional positive nonlinear system (23) is called absolutely stable if $x_{i} \in \mathbb{R}_{+}^{n}, i \in \mathbb{Z}_{+}$, and

$$
\lim _{i \rightarrow \infty} x_{i}=0, \quad \forall x_{0} \in \mathbb{R}_{+}^{n} .
$$

The matrix $A_{\alpha} \in \mathbb{R}_{+}^{n \times n}$ is called a Schur matrix if all its eigenvalues $z_{i}, i=1, \ldots, n$, satisfy the condition

$$
\left|z_{i}\right|<1, \quad i=1, \ldots, n .
$$

Theorem 8. The fractional positive nonlinear system (23) is absolutely stable if 
(i) $A_{\alpha} \in \mathbb{R}_{+}^{n \times n}$ is a Schur matrix and

$$
B \in \mathbb{R}_{+}^{n \times 1}, \quad C \in \mathbb{R}_{+}^{1 \times n}
$$

(ii) the nonlinear characteristic $f\left(e_{i}\right)$ satisfies the condition (29).

Proof. The proof is based on the Lyapunov method for fractional positive nonlinear systems. As a candidate for the Lyapunov function, the following linear function of the state vector $x_{i} \in \mathbb{R}_{+}^{n}, i \in \mathbb{Z}_{+}$, is chosen

$$
\begin{aligned}
V_{\alpha}\left(x_{i}\right) & =\lambda^{T} x_{i}, \\
\lambda^{T} & =\left[\begin{array}{lll}
\lambda_{1} & \ldots & \lambda_{n}
\end{array}\right], \\
\lambda_{k} & >0, \quad k=1, \ldots, n .
\end{aligned}
$$

Using (34) and (27a), we obtain

$$
\begin{aligned}
\Delta^{\alpha} V\left(x_{i}\right) & =V_{\alpha}\left(x_{i+1}\right)-V_{\alpha}\left(x_{i}\right) \\
& =\lambda^{T}\left[x_{i+1}-x_{i}\right] \\
& =\lambda^{T}\left[A_{\alpha}-I_{n}\right]+\lambda^{T} B f\left(e_{i}\right)<0
\end{aligned}
$$

since, by (11), we have

$$
\begin{aligned}
& B \in \mathbb{R}_{+}^{n \times 1}, \quad \lambda^{T}\left[A_{\alpha}-I_{n}\right]<0 \\
& B f\left(-e_{i}\right)<0, \quad i \in \mathbb{Z}_{+} .
\end{aligned}
$$

Therefore, the fractional positive nonlinear system (23) is absolutely stable.

Remark 2. The absolute stability of the fractional positive nonlinear system (23) is directly independent of the transfer function of its linear part.

Example 2. Consider the fractional nonlinear system (23) with $\alpha=0.5$ and

$$
A=\left[\begin{array}{ll}
0.1 & 0.2 \\
0.2 & 0.3
\end{array}\right], \quad B=\left[\begin{array}{l}
1 \\
2
\end{array}\right], \quad C=\left[\begin{array}{ll}
1 & 0
\end{array}\right]
$$

and the characteristic of the nonlinear element satisfying the condition (29). The matrix $A \in \mathbb{R}_{+}^{2 \times 2}$ (defined by (36)) is a Schur matrix since its characteristic polynomial

$$
\begin{aligned}
\operatorname{det}\left[I_{2}(z+1)-A_{\alpha}\right] & =\left|\begin{array}{cc}
z+0.4 & -0.2 \\
-0.2 & z+0.2
\end{array}\right| \\
& =z^{2}+0.6 z+0.04
\end{aligned}
$$

has positive coefficients. The same result can be obtained by using the condition (10) since, for $\lambda^{T}=\left[\begin{array}{ll}0.8 & 1\end{array}\right]$ and

$$
\left[A_{\alpha}-I_{2}\right]=\left[\begin{array}{cc}
-0.4 & 0.2 \\
0.2 & -0.2
\end{array}\right]
$$

we have

$$
\lambda^{T}\left[A_{\alpha}-I_{2}\right]=\left[\begin{array}{l}
-0.12 \\
-0.04
\end{array}\right]<0 .
$$

Therefore, by Theorem 8 the fractional positive nonlinear system with (37) is absolutely stable for all nonlinear characteristics satisfying the condition (24).

\section{Concluding remarks}

The positivity and absolute stability of a class of fractional nonlinear continuous-time and discrete-time systems have been addressed. Necessary and sufficient conditions for the positivity of the fractional nonlinear systems have been established (Theorems 5 and 7). Sufficient conditions for the absolute stability of fractional nonlinear systems have been also obtained (Theorems 6 and 8). The discussion has been illustrated by numerical examples. The presented results can be extended to multi-input multi-output nonlinear systems and nonlinear systems with different fractional orders.

\section{Acknowledgment}

This work was supported by the National Science Centre in Poland under the work no. 2017/27/B/ST7/02443.

\section{References}

Ait Rami M. and Tadeo F. (2007). Controller synthesis for positive linear systems with bounded controls, IEEE Transactions on Circuits and Systems 54(2): 151-155.

Bartosiewicz, Z. (2017). On controllability of positive nonlinear continuous-time systems, Proceedings of the Asian Control Conference (ASCC), Gold Coast, Australia, pp. 298-302.

Berman, A. and Plemmons, R.J. (1994). Nonnegative Matrices in the Mathematical Sciences, SIAM, Philadelphia, PA.

Busłowicz, M. (2008). Stability of linear continuous-time fractional order systems with delays of the retarded type, Bulletin of the Polish Academy of Sciences: Technical Sciences 56(4): 319-324.

Busłowicz, M. (2012). Stability analysis of continuous-time linear systems consisting of $n$ subsystems with different fractional orders, Bulletin of the Polish Academy of Sciences: Technical Sciences 60(2): 279-284.

Busłowicz, M. and Kaczorek, T. (2009). Simple conditions for practical stability of positive fractional discrete-time linear systems, International Journal of Applied Mathematics and Computer Science 19(2): 263-269, DOI: 10.2478/v10006-009-0022-6.

Farina, L. and Rinaldi, S. (2000). Positive Linear Systems: Theory and Applications, J. Wiley, New York, NY.

Kaczorek, T. (2002). Positive $1 D$ and $2 D$ Systems, Springer, London.

Kaczorek, T. (2010). Positive linear systems with different fractional orders, Bulletin of the Polish Academy of Sciences: Technical Sciences 58(3): 453-458.

Kaczorek, T. (2011a). Positive linear systems consisting of $n$ subsystems with different fractional orders, IEEE Transactions on Circuits and Systems 5897: 1203-1210.

Kaczorek, T. (2011b). Selected Problems of Fractional Systems Theory, Springer, Berlin. 
Kaczorek, T. (2014). Descriptor positive discrete-time and continuous-time nonlinear systems, Proceedings of SPIE 9290: 92902Q.

Kaczorek, T. (2015a). Analysis of positivity and stability of discrete-time and continuous-time nonlinear systems, Computational Problems of Electrical Engineering 5(1): 127-130.

Kaczorek, T. (2015b). Positivity and stability of discrete-time nonlinear systems, IEEE 2nd International Conference on Cybernetics, Gdynia, Poland, pp. 156-159.

Kaczorek, T. (2015c). Stability of fractional positive nonlinear systems, Archives of Control Sciences 25(4): 491-496.

Kaczorek, T. (2016). Analysis of positivity and stability of fractional discrete-time nonlinear systems, Bulletin of the Polish Academy of Sciences: Technical Sciences 64(3): 491-494.

Kaczorek, T. (2018). Decentralized stabilization of fractional positive descriptor continuous-time linear systems, International Journal of Applied Mathematics and Computer Science 28(1): 135-140, DOI: 10.2478/amcs-2018-0010.

Mitkowski, W. (2008). Dynamical properties of Metzler matrices, Bulletin of the Polish Academy of Sciences: Technical Sciences 56(4): 309-312.

Oldham, K.B. and Spanier, J. (1974). The Fractional Calculus, Academic Press, New York, NY.

Ortigueira M.D. (2011). Fractional Calculus for Scientists and Engineers, Springer, Berlin/Heidelberg.

Ostalczyk P. (2008). Epitome of the Fractional Calculus: Theory and Its Applications in Automatics, Lodz University of Technology Press, Łódź, (in Polish).

Podlubny, I. (1999). Fractional Differential Equations, Academic Press, San Diego, CA.

Polyak B.T. and Shcherbakov P.S. (2002a). Superstable linear control systems. I: Analysis, Automation and Remote Control 63(8): 1239-1254.

Polyak B.T. and Shcherbakov P.S. (2002b). Superstable Linear Control Systems, II: Design, Automation and Remote Control 63(11): 1745-1763.

Radwan, A.G., Soliman, A.M., Elwakil, A.S. and Sedeek, A. (2009). On the stability of linear systems with fractional-order elements, Chaos, Solitons and Fractals 40(5): 2317-2328.

Sajewski, Ł. (2017a). Decentralized stabilization of descriptor fractional positive continuous-time linear systems with delays, 22nd International Conference on Methods and Models in Automation and Robotics (MMAR), Międzyzdroje, Poland, pp. 482-487.
Sajewski Ł. (2017b). Stabilization of positive descriptor fractional discrete-time linear system with two different fractional orders by decentralized controller, Bulletin of the Polish Academy of Sciences: Technical Sciences 65(5): 709-714.

Solteiro Pires, E.J., Tenreiro Machado, J.A., Moura Oliveira, P.B. (2006), Functional dynamics in genetic algorithms, Workshop on Fractional Differentiation and Its Application, Porto, Portugal, 414-419.

Vinagre, B.M., Monje, C.A. and Calderon, A.J. (2002) Fractional order systems and fractional order control actions, Proceedings of the Conference on Decision and Control CDC'02, Las Vegas, NV, USA, pp. 2550-2554.

Zhang, J., Han, Z. Wu, H. and Hung, J. (2014). Robust stabilization of discrete-time positive switched systems with uncertainties and average dwell time switching, Circuits Systems and Signal Processing 33: 71-95.

Xiang-Jun, W., Zheng-Mao, W. and Jun-Guo, L. (2008). Stability analysis of a class of nonlinear fractional-order systems, IEEE Transactions on Circuits and Systems II: Express Briefs 55(11): 1178-1182.

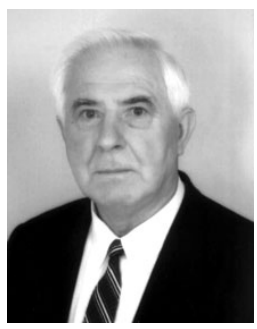

Tadeusz Kaczorek received his $\mathrm{MSc}, \mathrm{PhD}$ and DSc degrees in electrical engineering from the Warsaw University of Technology in 1956, 1962 and 1964, respectively. In the years 1968-1969 he was the dean of the Electrical Engineering Faculty, and in the period of 1970-1973 he was a deputy rector of the Warsaw University of Technology. In 1971 he became a professor and in 1974 a full professor at the same university. Since 2003 he has been a professor at the Białystok University of Technology. In 1986 he was elected a corresponding member and in 1996 a full member of the Polish Academy of Sciences. In the years 1988-1991 he was the director of the Research Center of the Polish Academy of Sciences in Rome. In 2004 he was elected an honorary member of the Hungarian Academy of Sciences. He was granted honorary doctorates by 13 universities. His research interests cover systems theory, especially singular multidimensional systems, positive multidimensional systems, singular positive 1D and 2D systems, as well as positive fractional 1D and 2D systems. He initiated research in the field of singular 2D, positive $2 \mathrm{D}$ and positive fractional linear systems. $\mathrm{He}$ published 28 books ( 8 in English) and over 1100 scientific papers. He also supervised $69 \mathrm{PhD}$ theses. He is the editor-in-chief of the Bulletin of the Polish Academy of Sciences: Technical Sciences and a member of editorial boards of ten international journals.

Received: 15 December 2017

Revised: 15 March 2018

Accepted: 5 June 2018 